\title{
Ecología
}

\section{Análisis trófico de Phymaturus punae (Iguania: Liolaemidae): variación estacional y sexual en la región más austral de la puna Argentina}

\author{
Trophic analysis of Phymaturus punae (Iguania: Liolaemidae): seasonal and sexual variation in the \\ most southern region of the Argentina Puna \\ Mariela Analía Córdoba ${ }^{a, b, *}$, Juan Carlos Acosta ${ }^{a}$, Héctor José Villavicencio ${ }^{a}$ \\ y Vanesa Astudillo ${ }^{a, b}$ \\ ${ }^{a}$ Departamento de Biología, Facultad de Ciencias Exactas, Físicas y Naturales, Universidad Nacional de San Juan. Av. Ignacio de la Roza 590, 5402, San Juan, \\ Argentina \\ ${ }^{\mathrm{b}}$ Consejo Nacional de Investigaciones Científicas y Técnicas, Av. Rivadavia 1917 - CABA, Buenos Aires, Argentina
}

Recibido el 13 de agosto de 2014; aceptado el 30 de junio de 2015

Disponible en Internet el 6 de noviembre de 2015

\begin{abstract}
Resumen
El conocimiento de los parámetros tróficos es de gran importancia para el entendimiento de las relaciones ecológicas entre las especies. Por ello, se describe y analiza la dieta de una población de Phymaturus punae en términos estacionales y en ambos sexos. Se analizaron los contenidos estomacales de 39 ejemplares calculando el porcentaje de cada categoría alimenticia. Se identificaron 16 categorías alimenticias y se agruparon luego en 4 más. Phymaturus punae es herbívoro, se alimenta principalmente de tallos y hojas, de individuos del género Adesmia. En diciembre, la categoría alimenticia más importante fue la de flores, y en febrero, la de tallos-hojas. La categoría más consumida por machos y hembras fue la de tallos-hojas. Se encontraron nemátodos en el $100 \%$ de la muestra.

Derechos Reservados @ 2015 Universidad Nacional Autónoma de México, Instituto de Biología. Este es un artículo de acceso abierto distribuido bajo los términos de la Licencia Creative Commons CC BY-NC-ND 4.0.
\end{abstract}

Palabras clave: Herbívoro; Nemátodos; Dieta; Categoría alimenticia

\section{Abstract}

Knowledge of trophic parameters is very important to understand ecological relationships between species. Therefore, we describe and analyze the diet of a Phymaturus punae population, at seasonal and sexual terms. We analyzed the stomach contents of 39 specimens and we calculated percentage of each food category. We identified 16 food categories, grouping then into another 4. Phymaturus punae is herbivorous, and feeds mainly on stems-leaves - from individuals of the genus Adesmia —. In December, the most important food category was flowers, whereas during February was stems-leaves. Either for males and females, stems-leaves was the most consumed category. Nematodes were found in $100 \%$ of the sample.

All Rights Reserved (C 2015 Universidad Nacional Autónoma de México, Instituto de Biología. This is an open access item distributed under the Creative Commons CC License BY-NC-ND 4.0.

Keywords: Herbivory; Nematodes; Diet; Food category

\footnotetext{
* Autor para correspondencia.

Correo electrónico: carranzamari@gmail.com (M.A. Córdoba).

La revisión por pares es responsabilidad de la Universidad Nacional Autónoma de México.
}

\section{Introducción}

El conocimiento de los parámetros tróficos es de gran importancia para la comprensión de las relaciones ecológicas entre las especies (Belver y Ávila, 2002). Los parámetros poblacionales relacionados con los hábitos alimentarios, el ciclo biológico y las 
estrategias en el uso del hábitat permiten evaluar objetivamente la vulnerabilidad de las especies y su estado de conservación, y suponen una información sustancial que debe tenerse en cuenta en la toma de decisiones de manejo (Aun y Martori, 1998; Christie, 1984; Martori, Juárez y Aun, 2002).

En general, la mayoría de los lagartos son insectívoros y a menudo oportunistas (Pianka, 1973), aunque en algunas especies cierta tendencia a la herbivoría en lagartos pequeños y de climas fríos (Espinoza, Wiens y Tracy, 2004). Pough (1973) postula que la herbivoría es común en reptiles de gran tamaño y peso superior a $100 \mathrm{~g}$. Sin embargo, en especies de Liolae$m u s$, de pequeñas dimensiones y pesos menores a los $100 \mathrm{~g}$, los vegetales constituyen una categoría alimenticia significativa (Quatrini, Albino y Barg, 2001). La herbivoría probablemente está favorecida en climas fríos debido a que los artrópodos son menos abundantes que las plantas "palatables" en hábitats de alta montaña y en latitudes altas (Pough et al., 2001).

Herbívoros y carnívoros se enfrentan a problemas dispares con respecto a la dieta y la digestión. La materia animal es fácilmente digerida y asimilada, mientras que en los vegetales la mayor parte de la energía está contenida en compuestos fibrosos, como la celulosa, para para cuya digestión los vertebrados carecen de enzimas. Por ello, los herbívoros poseen especializaciones intestinales ausentes en los carnívoros, tales como como ciego, válvas intestinales y la presencia de nemátodos en el intestino grueso (OǴrady, Morando, Ávila y Dearing, 2005), que facilitan la fermentación de componentes fibrosos y alargan el tiempo de retención de la digestión, lo que oncrementa la eficiencia digestiva (Iverson, 1982; Stevens, 1980; Stevens y Hume, 1998).

Phymaturus es un clado de lagartos herbívoros y vivíparos que se distribuye a partir del noroeste de Argentina, desde la provincia de Catamarca y a lo largo de los cordones montañosos de los Andes hasta la estepa patagónica y en áreas adyacentes a la cordillera de los Andes en Chile (Díaz-Gómez, 2008; Lobo, Abdala y Valdecantos, 2012). Tomando en cuenta las particularidades del género, las especies de Phymaturus han sido categorizadas recientemente como "vulnerables" (Abdala et al., 2012). Phymaturus punae muestra dimorfismo sexual representado por machos más grandes y robustos, aunque las hembras exhiben un cuerpo más amplio y una distancia entre los miembros anteriores y posteriores mayores (Boretto et al., 2007). Además, se observa un cambio en la coloración entre los lagartos que se encuentran dentro y fuera de las grietas de las rocas, siendo estos últimos más suaves, de color verde brillante y con un intenso melanismo solo en las cabezas de los machos, y los primeros presentan melanismo, sobre todo el cuerpo (Ibargüengoytía et al., 2008). Phymaturus punae alcanza una longitud hocico-cloaca de hasta $103.4 \mathrm{~mm}$ (Boretto, Fornés, Jahn, Acosta y Ibargüiengoytía, 2014), presenta hábitos saxícolas, propios de roquedales meteorizados, se encuentra por encima de los $3,500 \mathrm{~m}$, y se ha registrado en las localidades de Agua del Godo, Vega los Caserones, Agüita del Indio, Vega los Salineros II, Huesos quebrados, Ladera este del río San Guillermo, Llano Negro, Ladera oeste del río San Guillermo, Llano San Guillermo, Circuito Panorámico y Caserones (Acosta, Villavicencio y Marinero, 2007).
Es preferentemente herbívoro, como el resto de las especies del género (Cei, Etheridge y Videla, 1983), y presenta temperaturas corporales de actividad relativamente altas $\left(29-45^{\circ} \mathrm{C}\right)$, que logra por el comportamiento de asoleamiento en las crestas de los roquedales durante casi todo el tiempo de actividad diaria. El comportamiento de escape más frecuente es hacia las grietas de los roquedales graníticos, que también utiliza como madrigueras durante la inactividad (Ibargüengoytía et al., 2008). Phymaturus punae presenta un ciclo espermatogénico anual desde primavera hasta finales del verano. El ciclo de la hembras es bienal y está sincronizado con el de los machos, lo que indica que los ciclos bienales son una singularidad y carácter del género (Boretto et al., 2007, 2014). Los trabajos publicados sobre las características tróficas de especies del género Phymaturus en el oeste árido de Argentina son escasos (Acosta, Villavicencio y Blanco, 2008; Videla, 1983); el objetivo de este estudio fue analizar y describir la dieta determinando la existencia de variaciones estacionales, y entre ambos sexos, de una población de $P$. punae de la región más austral de la Puna Argentina, para aportar información de utilidad al estado de conservación de la especie.

\section{Materiales y métodos}

La Reserva de Biosfera San Guillermo ocupa una superficie total de 981,460 ha. Está conformada por 2 áreas protegidas de diferente jurisdicción, la Reserva Provincial y el Parque Nacional, homónimos. En la unidad de conservación se encuentran representados los biomas: Altoandino, Puna — porción austral— y Monte. Se ubica en el extremo noroeste de la provincia de San Juan, en los Andes centrales de Argentina, en el departamento de Iglesia. Está delimitada por la Cordillera de los Andes al oeste; la provincia de La Rioja al norte y este; el departamento Jáchal al este y el departamento Calingasta al sur. Geográficamente queda enmarcada por las siguientes coordenadas: $28^{\circ} 25^{\prime}-30^{\circ} 25^{\prime} \mathrm{S}$, $69^{\circ} 05^{\prime}-70^{\circ} 00^{\prime} \mathrm{O}$ (Martínez-Carretero et al., 2007). El clima de la región es árido-desértico, con grandes amplitudes térmicas diurnas-nocturnas y anuales, elevada heliofanía y escasa humedad. La temperatura media anual es de $6.7^{\circ} \mathrm{C}$; el mes más cálido es enero $\left(14^{\circ} \mathrm{C}\right)$ y el más frío julio $\left(-1^{\circ} \mathrm{C}\right)$ (Salvioli, 2007).

La distribución de las lluvias de la Reserva Provincial de San Guillermo presenta una alta heterogeneidad espacial. Hacia el oeste, de régimen mediterráneo, se registra la mayor incidencia de los frentes del Pacífico, y las lluvias alcanzan los 400-500 mm/año, principalmente como nieve o granizo; mientras que al este, con menores alturas, la influencia es Atlántica en el verano y escasa del Pacífico en invierno, y es una franja de tipo transicional con una incidencia de lluvias de $200 \mathrm{~mm} / \mathrm{año}$ (Cajal, Recay y Pujante, 1981).

La herpetofauna está representada por 5 especies de reptiles (Liolaemus eleodori, L. elongatus, L. ruibali, P. punae y Pristidactylus scapulatus) y una de anfibios (Chaunus spinulosus). Un estudio realizado por Cabrera y Monguillot (2006) ha dado a conocer una nueva especie: Liolaemus montanezi. Biológicamente el área es de sumo interés, dominando elementos del Monte en los niveles inferiores a los 2,500 m y de la Puna, además de Altoandinos entre los 3,000 m y el límite de la vegetación a 4,200-4,500 m. Las comunidades vegetales 
características están compuestas por Artemisia echegarayi, Lycium chanar, Fabiana punensis, Artemisia mendozana var. Paramilloensis y Larrea divaricata en suelos arenosos; de Lycium fuscum y Adesmia pinifolia en afloramientos saxícolas; de Stipa frigida, Adesmia subterranea, Jarava chrysophylla y J. speciosa var. Abscondita, y como especie endémica se encuentra Huarpea andina (Martínez-Carretero et al., 2007).

Phymaturus punae (Iguania: Liolaemidae) (Cei et al., 1983), es una especie endémica del Parque Nacional y de la Reserva Provincial San Guillermo. Ocupa prácticamente todos los roquedales del parque y la reserva, ya sea en cordones montañosos continuos, o bien, en islas o agrupamiento de rocas en los llanos y bolsones. Pertenece al grupo Palluma, subclado Puna y se encuentra emparentado con $P$. aguanegra y $P$. williamsi. El género de lagartos Phymaturus comprende un total de 36 especies de acuerdo con las publicaciones taxonómicas más recientes (Abdala y Quintero, 2014; Etheridge, 1995; Lobo y Quinteros, 2005; Lobo, Laspiur y Acosta, 2013).

Los ejemplares fueron capturados durante 2 periodos: del 5 al 9 de diciembre de 2004 y del 7 al 12 de febrero de 2005; las muestras se obtuvieron en el marco de un proyecto mayor destinado a conocer la reproducción, la morfometría, los ciclos hormonales y la termorregulación de la especie. Las capturas se realizaron mediante el método de lazo corredizo y a mano. Luego, los ejemplares fueron sacrificados mediante una inyección intraperitoneal de tiopental sódico, fijados al $10 \%$ con formaldehído por $24 \mathrm{~h}$ y, finalmente, preservados en etanol al 70\% (Aun y Martori, 1998; Boretto et al., 2007; Villavicencio, Acosta y Cánovas, 2005). Las muestras se depositaron en la colección científica herpetológica de la Universidad Nacional de San Juan (UNSJ).

En el laboratorio se procedió a la disección de los tractos gastrointestinales, colocando el material obtenido hidratado en cajas de Petri. Se realizó la identificación de las categorías alimenticias utilizando una lupa binocular estereoscópica (10-40×), un herbario de referencia de las especies del área de estudio, y cuando fue posible, y de acuerdo con el grado de digestión, se realizó la determinación taxonómica según Kiesling (1994, 2003), tratando de identificar la familia y el género. Para el recuento de las categorías alimenticias se consideró la abundancia de cada categoría, expresada como porcentaje de presencia en relación con el contenido total del tracto digestivo de cada individuo. Posteriormente estas se agruparon en 4 categorías alimenticias: 1) tallos-hojas, 2) semillas, 3) frutos y 4) flores.

Se realizó la cuantificación de la categoría alimenticia "frutos" y se estimó el volumen de cada uno de ellos utilizando la fórmula de Dunham (1983) de una esfera elipsoide:

$$
\mathrm{V}=4 / 3(1 / 2 \mathrm{~L}) *(1 / 2 \mathrm{~W})^{2}
$$

donde $\mathrm{L}=$ largo de la categoría alimenticia y $\mathrm{W}=$ ancho máximo de la categoría alimenticia. Para el resto de las categorías alimenticias se determinó solo la frecuencia de presencia que expresa la proporción de estómagos conteniendo una categoría específica. Para este caso, el número y el volumen no se pudieron determinar debido a que el material se encontraba muy fragmentado. Además, las semillas se observaron en grandes cantidades y su tamaño era reducido, lo que impidió su conteo. Para los nemátodos, arena, piedras, himenópteros y material no identificado se estimó el porcentaje de presencia en relación con el contenido total del tracto digestivo de cada individuo.

Los datos se trataron con estadísticos descriptivos: medias y desviación estándar $( \pm \mathrm{DE})$. Para asignar un hábito alimentario se utilizó el método de Espinoza et al. (2004), que se basa en el porcentaje de volumen de vegetales consumidos tomando en cuenta las siguientes definiciones: 0-10\%: insectívoro, 11-50\%: omnívoro y 51-100\%: herbívoro. Se realizó el test de Anova, el test a posteriori LSD de Fisher y el test de Student para analizar las variaciones en la composición de la dieta estacional y entre ambos sexos, haciendo pruebas individuales.

Para la categoría alimenticia "frutos", se calculó el índice de importancia relativa (IRI) (Pinkas, Oliphanat e Iverson, 1971):

$$
\mathrm{IRI}=\% \mathrm{FO}(\% \mathrm{~N}+\% \mathrm{~V})
$$

con el fin de determinar la contribución de dicha categoría en la dieta, donde $\% \mathrm{~N}$ expresa la importancia numérica, $\% \mathrm{~V}$ la importancia volumétrica y \%FO la proporción de estómagos que contienen una categoría específica. Se calculó el IRI para estimar la variación estacional y entre ambos sexos del material de la categoría alimenticia "frutos".

Se realizó un análisis global mediante el análisis de componentes principales (ACP), tomando como variables las categorías alimenticias: tallos-hojas, frutos, flores y semillas. Se realizó un análisis no paramétrico, mediante la prueba de Kruskal-Wallis con comparaciones de pares entre medias, para analizar diferencias entre las categorías alimenticias consumidas. Para los análisis estadísticos se utilizaron los programas InfoStat, versión 2.0 (Di Rienzo et al., 2008) y multivariate statistical package (MVSP) (Kovach, 2001), se consultó a Sokal y Rohlf (1999) de estadística paramétrica y Siegel (1980) de estadística no paramétrica.

\section{Resultados}

Del total de individuos, 38 se encontraron con el estómago lleno o semilleno, y solo uno tenía el estómago vacío, discriminados en 21 machos y 18 hembras. En total se encontraron 16 categorías alimenticias correspondientes a distintas estructuras o partes de vegetales (tabla 1). El porcentaje de volumen vegetal consumido por $P$. punae es del $82.14 \%$. Además, en el tracto digestivo se observó la presencia de nemátodos, himenópteros, arena, piedras y material no identificado, que se denominaron categorías no alimenticias (tabla 2). Se hallaron himenópteros en porcentajes mínimos, con un valor medio de $1.14( \pm \mathrm{DE}=0.80 ; \mathrm{N}=7)$; arena, con un valor medio de 1.02 $( \pm \mathrm{DE}=0.75 ; \mathrm{N}=9)$, y piedras, con un valor medio de 1.43 $( \pm \mathrm{DE}=0.81 ; \mathrm{N}=32)$. Los nemátodos se hallaron en el $100 \%$ de la muestra en el intestino grueso, con un valor medio de 12.36 $( \pm \mathrm{DE}=9.61 ; \mathrm{N}=38)$, el material no identificado debido al grado de digestión tuvo un valor medio de $9.40( \pm \mathrm{DE}=14.65 ; \mathrm{N}=32)$ (fig. 1). Para los valores medios se utilizaron solo aquellos estómagos en los que estaba presente la categoría no alimenticia.

En febrero se analizaron 25 ejemplares y se determinó que la categoría alimenticia más importante en cuanto al porcentaje del contenido estomacal fue "tallos-hojas" $=31 \%$, seguido de "frutos" $=8.2 \%\left(\right.$ Anova; $\left.\mathrm{F}_{(3 ; 56)}=16.32 ; \mathrm{N}=116 ; p<0.0001\right)$. 
Tabla 1

Categorías alimenticias consumidas en diciembre y febrero para ambos sexos de Phymaturus punae.

\begin{tabular}{|c|c|c|c|c|c|c|c|c|c|c|c|c|c|c|c|c|}
\hline \multirow[b]{3}{*}{ Categorías alimenticias } & \multicolumn{8}{|c|}{ Diciembre } & \multicolumn{8}{|c|}{ Febrero } \\
\hline & \multicolumn{4}{|c|}{ Machos } & \multicolumn{4}{|c|}{ Hembras } & \multicolumn{4}{|c|}{ Machos } & \multicolumn{4}{|c|}{ Hembras } \\
\hline & $\% \mathrm{FO}$ & $\% \mathrm{~N}$ & $\% \mathrm{Vol}$ & IRI & $\% \mathrm{FO}$ & $\% \mathrm{~N}$ & $\% \mathrm{Vol}$ & IRI & $\% \mathrm{FO}$ & $\% \mathrm{~N}$ & $\% \mathrm{Vol}$ & IRI & $\% \mathrm{FO}$ & $\% \mathrm{~N}$ & $\% \mathrm{Vol}$ & IRI \\
\hline $\begin{array}{l}\text { Adesmia tallos-hojas } \\
\text { Adesmia flor }\end{array}$ & 28.57 & & & & 16.7 & & & & $\begin{array}{l}50 \\
8.33\end{array}$ & & & & 38.46 & & & \\
\hline Lycium tallos-hojas & & & & & & & & & 25 & & & & 30.77 & & & \\
\hline Lecanophora & 14.29 & & & & & & & & 8.33 & & & & 7.69 & & & \\
\hline Ephedra tallos-hojas & & & & & & & & & 16.67 & & & & & & & \\
\hline Sphaeralcea & & & & & 16.7 & & & & 33.33 & & & & 30.77 & & & \\
\hline Portulacaceae & & & & & & & & & 25 & & & & 15.38 & & & \\
\hline Lycium frutos & & & & & & & & & 50 & 75.37 & 71 & $7,318.75$ & 15.38 & 75 & 70.59 & $2,239.82$ \\
\hline Ephedra frutos & 28.57 & 100 & 100 & $5,717.09$ & 16.7 & 100 & 100 & $3,333.33$ & 8.33 & 24.63 & 29 & 446.88 & 7.69 & 25 & 29.41 & 418.55 \\
\hline Descurania & 28.57 & & & & 16.7 & & & & 16.67 & & & & 15.38 & & & \\
\hline Cerastium & 14.29 & & & & & & & & & & & & & & & \\
\hline Polygala & 14.29 & & & & 16.7 & & & & & & & & & & & \\
\hline Primordios de Lycium & 28.57 & & & & & & & & 8.33 & & & & & & & \\
\hline Flores & 14.29 & & & & 33.3 & & & & & & & & 7.69 & & & \\
\hline Semillas & & & & & 33.3 & & & & 25 & & & & 15.38 & & & \\
\hline Tallos-hojas & 42.86 & & & & 16.7 & & & & 16.67 & & & & 15.38 & & & \\
\hline
\end{tabular}

$\% \mathrm{~N}$ : porcentaje de numerosidad en el total de las presas; \%FO: porcentaje de frecuencia de presencia en el total de estómagos; \% Vol: porcentaje volumétrico de las presas; IRI: índice de importancia relativa. 


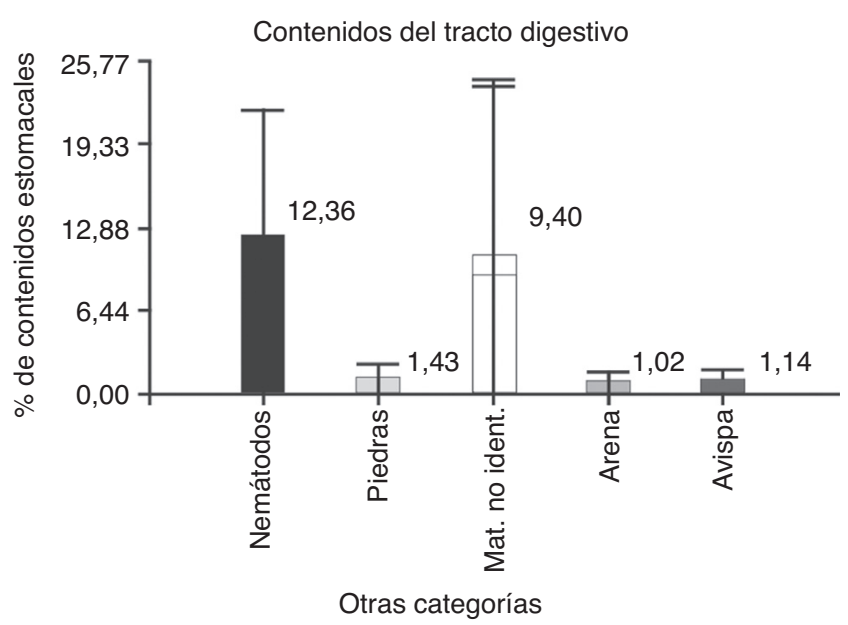

Figura 1. Porcentaje de contenidos estomacales pertenecientes a categorías no alimenticias halladas en los tractos digestivos de $P$. punae, para el total de la muestra.

Tabla 2

Características de otras categorías, presentes en los tractos digestivos de Phymaturus punae.

\begin{tabular}{ll}
\hline Otras categorías & Características de las categorías presentes \\
\hline Material no identificado & $\begin{array}{l}\text { Restos vegetales (tallos, hojas, flores, etc.) } \\
\text { no reconocidos debido al grado de digestión }\end{array}$ \\
Nemátodos & $\begin{array}{l}\text { Aproximadamente } 2 \text { a } 5 \mathrm{~mm} \\
\text { Pproximadamente entre } 2 \text { y } 5.5 \mathrm{~mm}\end{array}$ \\
Aredras & $\begin{array}{l}\text { Partículas }<1 \mathrm{~mm} \\
\text { Himenópteros }\end{array}$ \\
& $\begin{array}{l}\text { Se hallaron cabezas, tórax, alas y patas, } \\
\text { o partes de estas estructuras }\end{array}$ \\
\end{tabular}

El análisis a posteriori indicó un mayor consumo de tallos-hojas respecto a flores, semillas y frutos (fig. 2). Se encontraron nemátodos en el $100 \%$ de la muestra, con un valor medio de 13.46 $( \pm \mathrm{DE}=10.63 ; \mathrm{N}=25)$; también se observaron piedras, arena y material vegetal no identificado por su grado de digestión.

En diciembre se analizaron 13 ejemplares, y la categoría alimenticia más importante en cuanto al porcentaje de contenidos estomacales fue "flores" $=15.8 \%$, seguidos de "tallos-hojas" $=14.8 \%$ (fig. 2). No hubo diferencias en las categorías alimenticias consumidas en el mes de diciembre (Anova; $\mathrm{F}_{(3 ; 72)}=1.99 ; \mathrm{N}=60 ; p=0.12$ ) (fig. 2). Cabe destacar que se encontraron nemátodos en el $100 \%$ de la muestra, con un valor medio de $10.31( \pm \mathrm{DE}=7.24 ; \mathrm{N}=13)$, así como piedras, arena y material vegetal no identificado por su estado de digestión.

$\mathrm{Al}$ comparar las estaciones se observó que el consumo de tallos-hojas fue mayor en febrero que en diciembre ( $t$ de Student $=4.79 ; \mathrm{N}=13 ; p<0.0001$ ). Por otro lado, entre diciembre $\mathrm{y}$ febrero no hubo diferencias en cuanto al consumo de las categorías "flores" ( $\mathrm{t}$ de Student $=1.77 ; \mathrm{N}=13 ; p=0.09$ ), "frutos" (t de Student $=0.21 ; \mathrm{N}=13 ; p=0.21)$ y "semillas" ( $\mathrm{t}$ de Student $=0.23 ; \mathrm{N}=13 ; p=0.82$ ). No hubo diferencias significativas entre las 16 categorías alimenticias consumidas en diciembre (Kruskal-Wallis, $\mathrm{H}=17.04 ; \mathrm{N}=30 ; p=0.19$ ), pero sí en febrero (Kruskal-Wallis, $\mathrm{H}=43.11 ; \mathrm{N}=74 ; p<0.0001$ ). Del análisis a posteriori podemos observar que en febrero hubo un

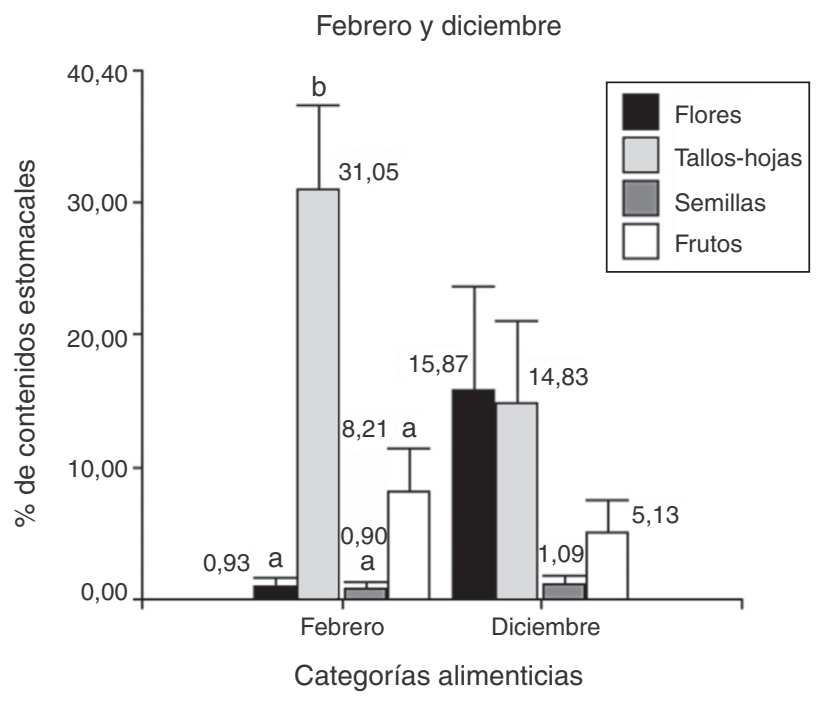

Figura 2. Porcentaje de los contenidos estomacales de $P$. punae para febrero y diciembre de las 4 categorías alimenticias: flores, tallos-hojas, semillas y frutos. La prueba realizada para febrero indica diferencias significativas (LSD de Fisher, $p \leq 0.05$, letras diferentes indican diferencias estadísticas). La prueba realizada para diciembre no detecta significación estadística (ver texto).

mayor consumo de Adesmia, tallos-hojas respecto a Descurania, semillas, y Portulacacea (fig. 3).

De 39 ejemplares, 18 corresponden a hembras y 21 a machos, y la categoría alimenticia más importante en cuanto al porcentaje de contenidos estomacales fue "tallos-hojas" (hembras = $23.4 \%$ y machos $=29.8 \%$ ), seguida de "frutos" (hembras $=2.8 \%$ y machos $=12 \%$ ). Se hallaron diferencias significativas entre las categorías alimenticias consumidas por las hembras (Anova; $\mathrm{F}_{(3 ; 68)}=6.95 ; \mathrm{N}=72 ; p=0.0004$ ) y los machos (Anova; $\left.\mathrm{F}_{(3 ; 80)}=6.97 ; \mathrm{N}=84 ; p=0.0003\right)$. Del análisis a posteriori se observa que la categoría alimenticia más consumida en ambos sexos fue "tallos-hojas" (fig. 4). Se encontraron nemátodos en el $100 \%$ de las muestras, además de piedras, arena y material vegetal no identificado por su estado de digestión.

Se analizaron 38 ejemplares de $P$. punae. El consumo de frutos fue mayor en machos ( $\mathrm{t}$ de Student $=2.15 ; \mathrm{N}_{\text {hembras }}=18$; $\left.\mathrm{N}_{\text {machos }}=21 ; p=0.04\right)$. Sin embargo, no se detectaron diferencias significativas entre ambos sexos respecto a las flores $(\mathrm{t}$ de Student $=1.56 ; \mathrm{N}_{\text {hembras }}=18 ; \mathrm{N}_{\text {machos }}=21 ; p=0.13$ ), semillas $\left(\mathrm{t}\right.$ de Student $\left.=-0.70 ; \mathrm{N}_{\text {hembras }}=18 ; \mathrm{N}_{\text {machos }}=21 ; p=0.48\right) \mathrm{o}$ tallos-hojas (t de Student $=-1.01 ; \mathrm{N}_{\text {hembras }}=18 ; \mathrm{N}_{\text {machos }}=21$; $p=0.31$ ). Se hallaron diferencias significativas entre las 16 categorías consumidas en las hembras (Kruskal-Wallis, $\mathrm{H}=30.08$; $\mathrm{N}=42 ; \quad p=0.0014$ ) y en los machos (Kruskal-Wallis, $\mathrm{H}=30.92 ; \mathrm{N}=52 ; p=0.0129)$. En los hembras, Adesmia talloshojas fueron más consumidas que semillas (prueba LSD de Fisher) (fig. 6). En machos, Adesmia tallos-hojas fueron más consumidas que las semillas, Portulacacea, Descurania, primordios de Lycium y tallos-hojas (fig. 5).

De las 16 categorías alimenticias, en diciembre se encontró que la categoría alimenticia fundamental fue Ephedra frutos $(\%$ IRI $=100 \%)$ en ambos sexos, mientras que en febrero la categoría alimenticia fundamental fue Lycium frutos (\%IRI = 100\%) en ambos sexos, y Ephedra frutos fue accidental (machos 


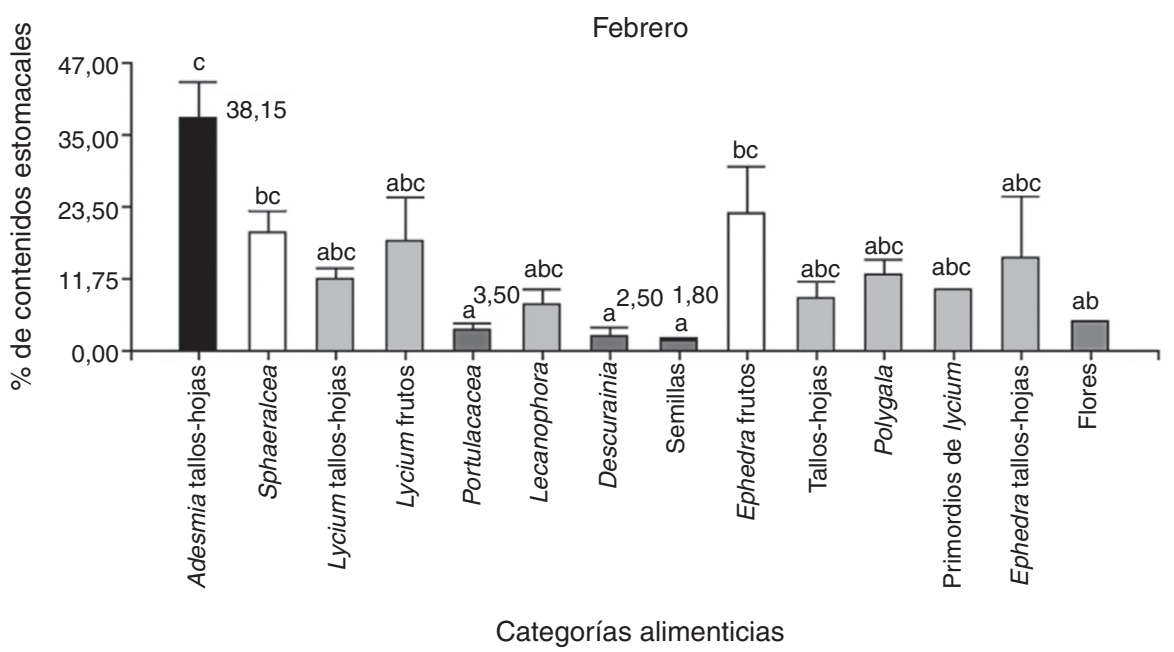

Figura 3. Porcentaje de contenidos estomacales de P. punae, obtenidos para las 14 categorías alimenticias consumidas en el mes de febrero. Letras diferentes indican diferencias estadísticas significativas según prueba LSD de Fisher $(\alpha=0.05)$.

$\% \mathrm{IRI}=6.11 \%$ y hembras $\% \mathrm{IRI}=18.69 \%$ ). Con respecto al $\%$ Vol, para febrero Lycium frutos fue la categoría alimenticia de mayor contribución en ambos sexos, mientras que en diciembre fue Ephedra frutos (tabla 1). La categoría predominante en términos de $\% \mathrm{FO}$ para diciembre fue tallos-hojas $(\% \mathrm{FO}=$ 42.86) en machos y para hembras fue flores y semillas $(\% \mathrm{FO}=33.33)$; en febrero los machos presentaron una mayor frecuencia de las categorías alimenticias Lycium frutos y Adesmia tallos-hojas $(\% \mathrm{FO}=50 \%)$ y en hembras Adesmia tallos-hojas $(\% \mathrm{FO}=38.46)$ (tabla 1$)$.

No se observó correlación entre las variables tallos-hojas y semillas, flores y frutos. Asimismo, la variable frutos no se correlacionó con semillas, flores y tallos-hojas. Sin embargo, se puede observar que existe una fuerte correlación entre las variables semillas y flores. La componente (CP1) explica el $32.1 \%$ de la variabilidad y separa las variables tallos-hojas y

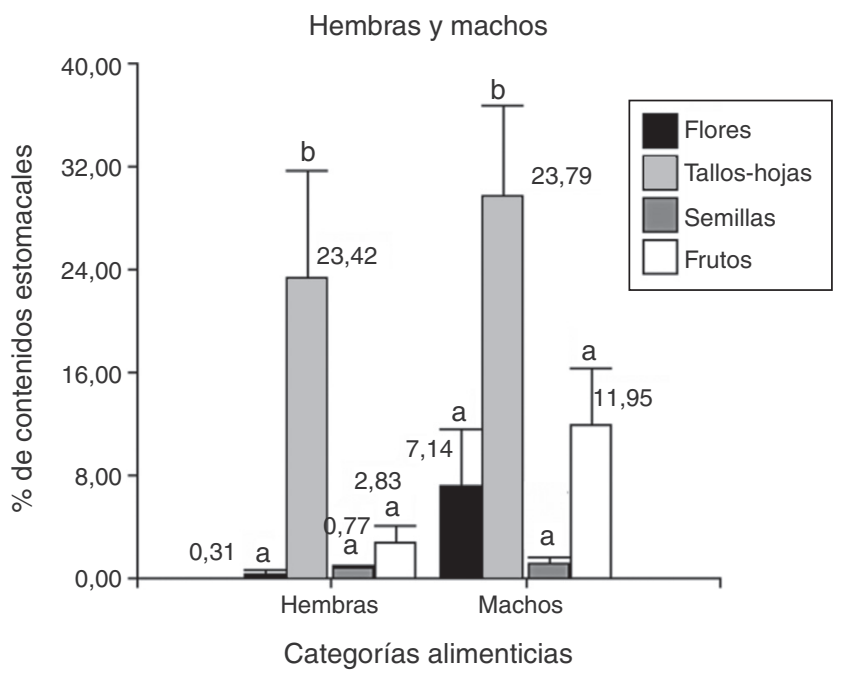

Figura 4. Porcentaje de los contenidos estomacales de P. punae para machos y hembras de las 4 categorías alimenticias: flores, tallos-hojas, semillas y frutos. Ambas pruebas realizadas en machos y en hembras, detectan diferencias estadísticas significativas $(\alpha=0.05)$ que se indican con letras diferentes. frutos de las variables semillas y flores. Las flores y las semillas son consumidas mayormente en diciembre y muy poco en febrero (fig. 7). Sin embargo, el consumo de tallos-hojas y frutos es mayor en febrero. Con los 2 primeros componentes es posible explicar el $57 \%$ de la variación total. Para la CP1, tallos-hojas recibe el peso negativo más alto y flores el peso positivo más alto — variabilidad de los hábitos alimentarios por ingesta-. Se observó que los machos consumían flores, semillas y frutos, y las hembras presentaban un elevado consumo de tallos-hojas y un menor consumo de frutos, sin observarse consumo de flores y semillas (fig. 8).

\section{Discusión}

Phymaturus punae es una especie herbívora que basa su dieta en el consumo de tallos-hojas del género Adesmia, Licyum y Ephedra, y el resto de las categorías alimenticias se encuentran como: Adesmia flores; Lecanophora, Sphaeralcea, Portulacaceae, Lycium frutos; Ephedra frutos; Descurania, Cerastium, Polygala primordios de Lycium flores, semillas y tallos-hojas, en menores porcentajes; mientras que $P$. aff. palluma, de distribución andino-patagónica, consume principalmente frutos de Berberis empetrifolia, Rumex acetosella y Calandrinia sp., detectados por la presencia de semillas en las heces fecales (Celedón-Neghme, Salgado y Victoriano, 2005). Asimismo, en una población de $P$. antofagastensis en la cordillera de Catamarca, los frutos de L. chanar y estróbilos de Ephedra multiflora fueron las categorías principales de la dieta (Acosta et al., 2008). A diferencia de $P$. cf. palluma de los Andes centrales en Argentina, que presenta una alimentación basada en flores de Adesmia pinnifolia (Castro, Laspiur y Acosta, 2013). Por otra parte, en Chile, en la Reserva Nacional Altos de Lircay (El Enladrillado), $P$. flagellifer consume sobre todo hojas, flores y frutos de Adesmia aff. hirsuta según su disponibilidad durante el periodo estival (Araya-Díaz, 2007). También una población de P. palluma en Mendoza consume principalmente plantas de los géneros Artemisia y Ephedra (Videla, 1983). Además, Liolaemus poecilochromus de los Andes del noroeste en Argentina es 


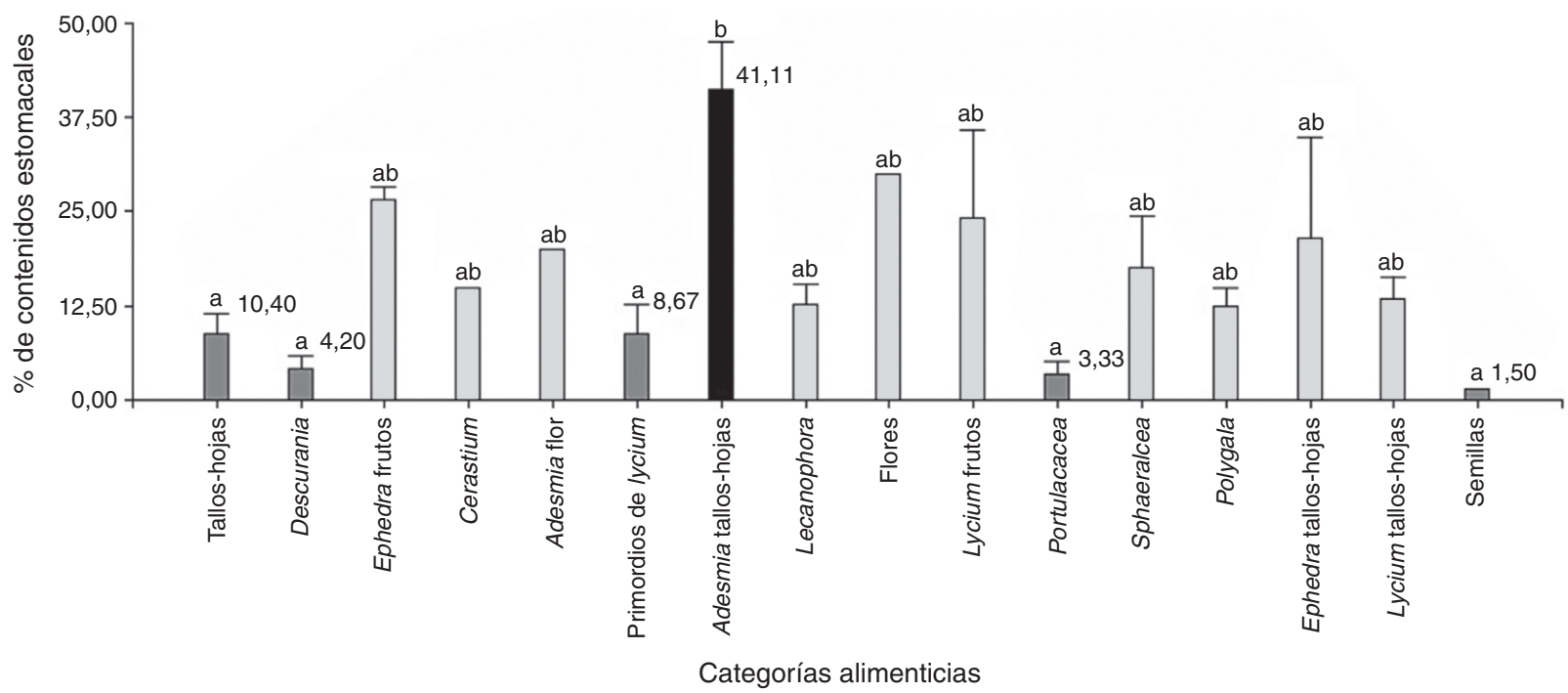

Figura 5. Porcentaje de los contenidos estomacales, obtenidos para las 17 categorías alimenticias de $P$. punae para machos. Letras diferentes indican diferencias estadísticas significativas según prueba LSD de Fisher $(\alpha=0.05)$.

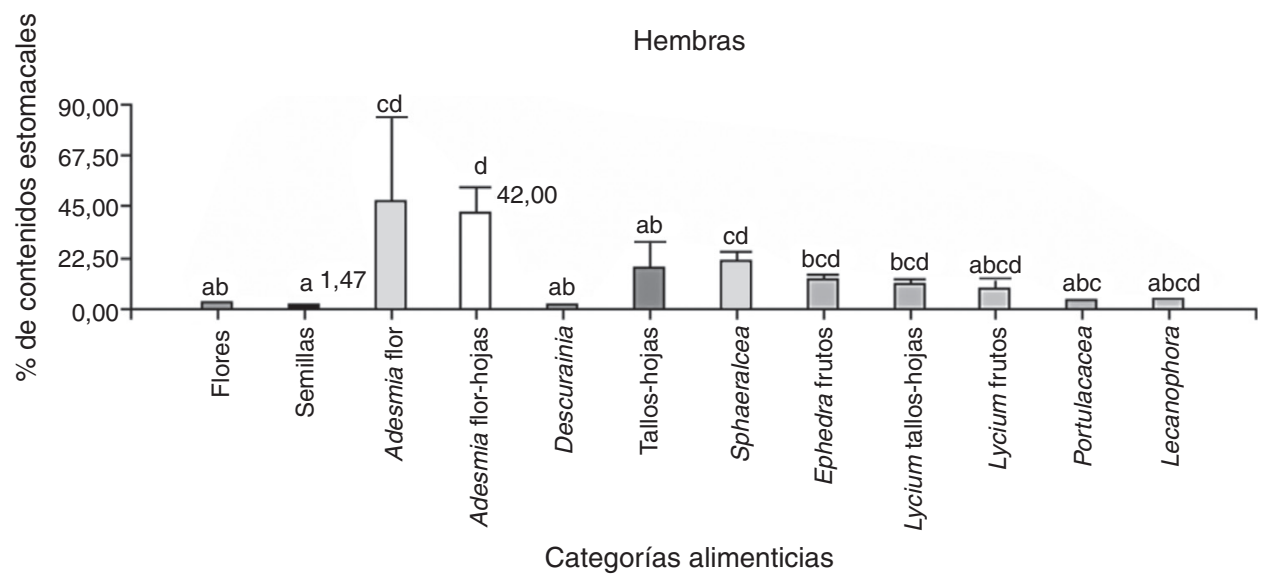

Figura 6. Porcentaje de los contenidos estomacales de P. punae, obtenidos para las 12 categorías alimenticias consumidas por las hembras. Letras diferentes indican diferencias estadísticas significativas, según prueba LSD de Fisher $(\alpha=0.05)$.

principalmente herbívoro ( $80 \%$ de plantas en la dieta), incluso en los juveniles, lo que lo convierte en el reptil herbívoro más pequeño conocido (Valdecantos, Arias y Espinoza, 2012).

La presencia de arena y piedras en los contenidos estomacales de $P$. punae puede deberse a una ingesta accidental o a la participación en la digestión mecánica, como se ha sugerido previamente. En un estudio sobre la dieta de Liolaemus wiegmannii, Aun, Martori y Rocha (1999) sugieren que estos elementos podrían intervenir en la digestión mecánica de los alimentos. Este fenómeno se ha observado además en Liolaemus elongatus, Liolaemus ruibali (Videla, 1983; Villavicencio et al., 2005) y Liolaemus quilmes (De Viana, Jovanovich y Valdés, 1994). Por las cantidades encontradas en los tractos digestivos se sugiere un consumo accidental o involuntario.

De la misma forma, la baja frecuencia de aparición en los contenidos estomacales sugiere que el consumo de insectos se debe a una ingesta accidental, como consecuencia de la estrecha asociación insectos-flores. Por otra parte, la herbivoría probablemente

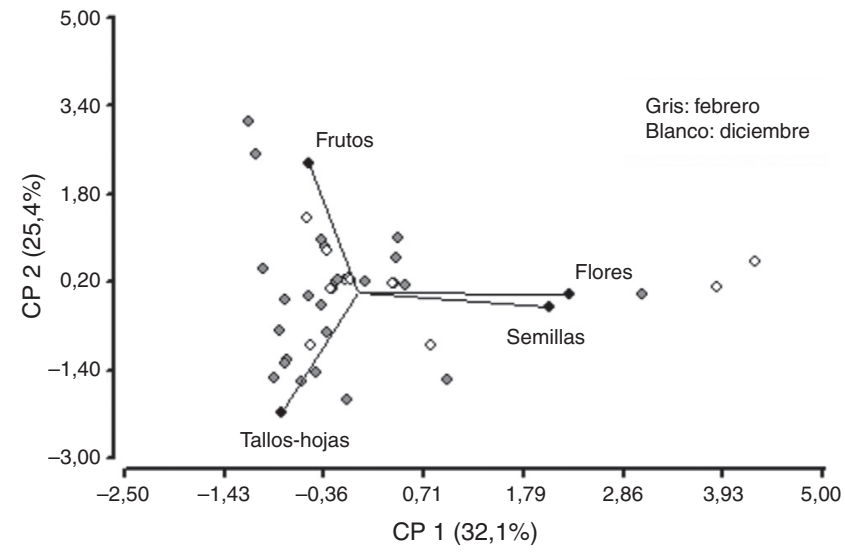

Figura 7. Gráfico bidimensional para la variabilidad de las 4 categorías alimenticias: frutos, flores, semillas y tallos-hojas, mediante el análisis de componentes principales (ACP) con 2 componentes que explican el 57\% de la varianza total, para la distribución de las variables para diciembre y febrero. 


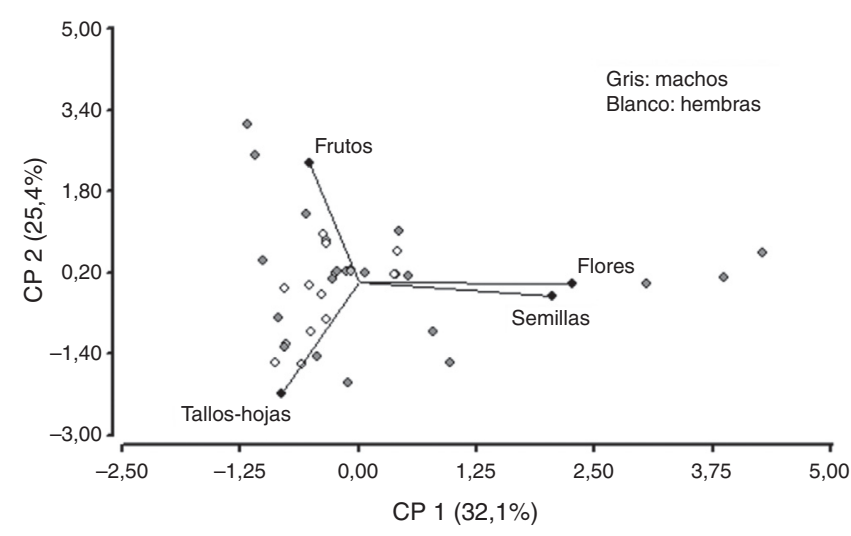

Figura 8. Variabilidad de las 4 categorías alimenticias: frutos, flores, semillas y tallos-hojas, mediante el análisis de componentes principales (ACP) con 2 componentes que explican el $57 \%$ de la varianza total, para la distribución de las variables para machos y hembras.

es favorecida en ambientes de alta montaña, con grandes variaciones en las temperaturas entre el día y la noche, debido a que los insectos, como recurso alimenticio, son efímeros y menos abundantes en comparación con la disponibilidad de plantas en hábitats de alta montaña (Espinoza et al., 2004).

Cabe destacar que la presencia de nemátodos en el 100\% de los individuos analizados podría estar relacionada con el tipo de dieta herbívora. Los nemátodos en el intestino grueso, de hecho, pueden ser parásitos, pero su gran incidencia en reptiles herbívoros y las altas densidades en las que se encuentran dentro de un solo animal sugieren que los nemátodos del intestino grueso pueden ayudar a los reptiles herbívoros en la digestión del material vegetal (Iverson, 1982). Así, OǴrady, Morando, Ávila y Dearing (2005) sugieren que los nemátodos en el intestino grueso desempeñan un rol en la digestión y asimilación del material vegetal. Además, el intestino grueso de los herbívoros es más largo y más voluminoso que el de carnívoros de tamaño similar (Iverson, 1982).

Pough (1973) y Vitt (2004) postulan que la herbivoría ha surgido en especies de reptiles de gran tamaño y distribuidas en regiones de clima cálido. Sin embargo, la herbivoría en lagartos pequeños se ha documentado en Argentina, en lagartos que habitan principalmente en ambientes fríos (Castro et al., 2013; Quatrini et al., 2001; Valdecantos et al., 2012). Pough (1973) sostiene que la herbivoría es compatible con tamaños corporales superiores a $100 \mathrm{~g}$; sin embargo, O'Grady et al. (2005) informan que existen lagartos herbívoros de la familia Liolaemidae que presentan una masa corporal de 30-80 g. Esto coincide con lo citado por Valdecantos et al. (2012) para L. poecilochromus, el cual presenta una masa corporal de $10 \mathrm{~g}$, y con lo observado en este estudio para $P$. punae, con una masa corporal de $23 \mathrm{~g}$. Además, el consumo de estructuras vegetales se ha observado en otras especies de pequeño tamaño de la familia, como L. ruibali, que consume voluntariamente frutos de L. chanar, en Liolaemus bibroni (Belver y Ávila, 2002) donde el 48\% de los estómagos contenían material vegetal, y en L. wiegmannii (Aun et al., 1999) donde también se han observado componentes vegetales.

Phymaturus punae solo presenta una variación significativa en su dieta en el mes de febrero, donde la categoría principal fue tallos-hojas, y para el mes de diciembre en que fue flores seguido de tallos-hojas. Sazima, Sazima y Sazima (2005) sugieren que los lagartos que comen flores tienen un doble propósito: la adquisición de energía y el balance hídrico.

En este estudio, machos y hembras exhiben diferencias en el consumo de algunas categorías alimenticias. Los machos consumen principalmente frutos, flores y semillas, y las hembras presentan un elevado consumo de tallos-hojas y un menor consumo de frutos. Existen revisiones que argumentan que los lagartos pertenecientes a la familia Iguanidae consumen principalmente las partes reproductivas de las plantas, como flores y/o frutos (Godínez-Álvarez, 2004). Esto coincide con lo registrado por Dutra, Siqueira, Vrcibradic, Kiefer y Rocha (2011) quienes establecen, para lagartos tropicales herbívoros, que las flores y los frutos son las principales partes de la planta que se consumen, probablemente porque contienen menos material fibroso y celulosa que las estructuras verdes y, por lo tanto, se digieren y asimilan más fácilmente. Esto justificaría lo observado en $P$. punae, ya que generalmente los machos de Iguania tienen mayor área de acción que las hembras (Perry y Garland, 2002); de esta manera, los machos podrían tener la posibilidad de seleccionar más variedad y cantidad de categorías alimenticias para su consumo que las hembras que consumen principalmente tallos-hojas.

Según el índice de importancia relativa en la categoría alimenticia frutos, se observan diferencias estacionales, pero no entre ambos sexos. Estas diferencias estacionales, en las cuales se encuentra a Ephedra frutos como categoría fundamental en diciembre y Lycium frutos en febrero, podrían coincidir con épocas de mayor disponibilidad y oferta de alimento; además, a nivel fenológico, son épocas de floración y fructificación de las distintas especies vegetales.

Este estudio permite conocer un aspecto importante de la ecología de la especie que habita en ambientes rigurosos como son los de alta montaña, y es el primero que aporta información sobre la alimentación de esta, con lo cual se establecen bases para estudios posteriores referidos tanto a uso de otras dimensiones del nicho ecológico de $P$. punae como a sus relaciones intra e interespecíficas, todos aspectos fundamentales que contribuyen a la conservación de la especie.

Puede concluirse que Phymaturs punae es una especie herbívora, que se alimenta principalmente de tallos-hojas (del género Adesmia, Lycium y Ephedra), seguido de frutos (del género Lycium y Ephedra), flores y semillas, y muestra variaciones estacionales, pero no entre ambos sexos; así, los lagartos pueden seleccionar plantas que les permitan obtener una dieta balanceada (Rocha, 2000) y, a su vez, contribuir y ser esenciales en la reproducción de algunas plantas y participar en los procesos de polinización y dispersión de semillas de manera exitosa (Godínez-Álvarez, 2004; Galindo-Uribe y Hoyos-Hoyos, 2007; Olesen y Valido, 2003).

\section{Agradecimientos}

Agradecemos a la Subsecretaría de Medio Ambiente de la provincia de San Juan por los permisos autorizados para realizar la recolecta de la muestra. A Antonio Dalmasso y Justo 
Márquez por su ayuda en la identificación de especies vegetales. A José Rogelio Cedeño-Vázquez por su asistencia en el proceso editorial del manuscrito y a los revisores anónimos por sus valiosos comentarios. Este estudio fue apoyado por la Universidad Nacional de San Juan.

\section{Referencias}

Abdala, C. S., Acosta, J. C., Acosta, J. L., Álvarez, B. B., Arias, F., Ávila, L. J., et al. (2012). Categorización del estado de conservación de las lagartijas y anfisbenas de la República Argentina. Cuadernos de Herpetología, 26, 215-248.

Abdala, C. S. y Quintero, A. S. (2014). Los últimos 30 años de estudios de la familia de lagartijas más diversa de Argentina. Actualización taxonómica y sistemática de Liolaemidae. Cuadernos de Herpetología, 28, 55-82.

Acosta, J. C., Villavicencio, H. J. y Blanco, G. M. (2008). Phymaturus antofagastensis (NCN) Diet. Herpetological Review, 39, 91.

Acosta, J. C., Villavicencio, H. J. y Marinero, J. A. (2007). Anfibios y reptiles. Biodiversidad, bio-ecología y especies de valor especial para monitoreo. En E. Martínez-Carretero (Ed.), Diversidad biológica y cultural de los Altos Andes Centrales de la Argentina: línea de base de la Reserva de la Biosfera San Guillermo (pp. 167-179). San Juan: Universidad Nacional de San Juan.

Araya-Díaz, S. (2007). Aspectos autecológicos relevantes para la conservación de Phymaturus flagellifer (Reptilia: Tropiduridae) en la Reserva Nacional Altos de Lircay, región del Maule. Tesis. Facultad de Ciencias Agronómicas Escuela de Agronomía, Universidad de Chile.

Aun, L. y Martori, R. (1998). Reproducción y dieta de Liolaemus koslowskyi Etheridge 1993. Cuadernos de Herpetología, 12, 1-9.

Aun, L., Martori, R. y Rocha, C. (1999). Variación estacional de la dieta de Liolaemus wiegmannii (Squamata: Tropiduridae) en un Agroecosistema del Sur de Córdoba. Argentina. Cuadernos de Herpetología, 13, 69-80.

Belver, L. C. y Ávila, L. J. (2002). Diet composition on Liolaemus bibronii (Iguania: Liolaemidae) in Southern río Negro Province, Argentina. Herpetological Journal, 12, 39-42.

Boretto, J. M., Fornés, M. W., Jahn, G. A., Acosta, J. C. y Ibargüengoytía, N. R. (2014). Testosterone cycle and regulation of reproductive events in the lizard Phymaturus punae (Liolaemidae) from the highlands of the Andes, Argentina. Journal of Herpetology, 48, 172-180.

Boretto, J. M., Ibargüengoytía, R. N., Acosta, J. C., Blanco, G. M., Villavicencio, J. y Marinero, J. A. (2007). Reproductive biology and sexual dimorphism of a high-altitude population of the viviparous lizard Phymaturus punae from the Andes in Argentina. Amphibia-Reptilia, 28, 427-432.

Cabrera, R. C. y Monguillot, J. C. (2006). A new Andean species of Liolaemus of the darwinii complex (Reptilia: Iguanidae). Zootaxa, 1106, 35-43.

Cajal, J. L., Recay, A. A. y Pujante, J. C. (1981). La Reserva Provincial San Guillermo y sus asociaciones ambientales. Buenos Aires: SECYT, Ministerio de Cultura y Educación.

Castro, S. A., Laspiur, A. y Acosta, J. C. (2013). Variación anual e intrapoblacional de la dieta de Phymaturus cf. palluma (Iguania: Liolaemidae) de los Andes centrales en Argentina. Revista Mexicana de Biodiversidad, 84, $1258-1265$.

Cei, J. M., Etheridge, R. y Videla, F. (1983). Especies nuevas de Iguánidos del noroeste de la Provincia de San Juan (Reserva Provincial San Guillermo), Argentina. Deserta, 7, 316-323.

Celedón-Neghme, C., Salgado, C. R. y Victoriano, P. F. (2005). Preferencias alimentarias y potencial dispersor del lagarto herbívoro Phymaturus flagellifer (Tropiduridae) en los Andes. Gayana, 69, 266-276.

Christie, M. I. (1984). Determinación de las prioridades de conservacionistas, para la fauna de vertebrados patagónicos. Revista del Museo Argentino de Ciencias Naturales. Buenos Aires, Zoología, 13, 535-539.

De Viana, M. L., Jovanovich, C. y Valdés, P. (1994). Hábitos alimentarios de Liolaemus darwinii (Sauria: Iguanidae), en el Valle de TinTin, Argentina. Revista de Biología Tropical, 42, 379-381.

Di Rienzo, J. A., Casanoves, F., Balzarini, M. G., González, L., Tablada, M. y Robledo, C. W. (2008). InfoStat. Versión 2008, Grupo InfoStat, FCA. Córdoba: Universidad Nacional de Córdoba.
Díaz-Gómez, J. M. (2008). Historical biogeography of Phymaturus (Iguania: Liolaemidae) from Andean and Patagonian South American. Zoologica Scripta, 38, 1-7.

Dunham, A. E. (1983). Realized niche overlap, resource abundance, and intensity of interspecific competition. En R. B. Huey, E. R. Pianka y T. W. Schoener (Eds.), Lizard ecology, studies of a model organism (pp. 61-280). Cambridge, Massachusetts: Harvard University Press.

Dutra, G. F., Siqueira, C. C., Vrcibradic, D., Kiefer, M. C. y Rocha, C. F. (2011). Plant consumption of insular and mainland populations of a tropical lizard. Herpetológica, 67, 32-45.

Espinoza, R. E., Wiens, J. J. y Tracy, R. C. (2004). Recurrent evolution of herbivory in small, cold-climate lizards: breaking the ecophysiological rules of reptilian herbivory. Proceedings of the National Academy of Sciences, 101, 16819-16824.

Etheridge, R. E. (1995). Redescription of Ctenoblepharys adspersa Tschudi, 1845, and the taxonomy of Liolaeminae (Reptilia: Squamata: Tropiduridae). American Museum Novitates, 3142, 1-34.

Galindo-Uribe, D. y Hoyos-Hoyos, J. M. (2007). Relaciones plantaherpetofauna: nuevas perspectivas para la investigación en Colombia. Revista de la Facultad de Ciencias, 12, 9-34.

Godínez-Álvarez, H. (2004). Pollination and seed dispersal by lizards: a review. Revista Chilena de Historia Natural, 77, 569-577.

Ibargüengoytía, N. R., Acosta, J. C., Boretto, J. M., Villavicencio, H. J., Marinero, J. A. y Krenz, J. D. (2008). Thermal biology of Phymaturus lizards: comparisons from the Andes to the Patagonian steppe in Argentina. Journal of Arid Environments, 72, 1620.

Iverson, J. B. (1982). Adaptations to herbivory in Iguanine lizards. En G. M. B. Rand y A. S. Rand (Eds.), Iguanas of the world: their behavior, ecology, and conservation (pp. 60-76). Park Ridge: Noyes Publications.

Kiesling, R. (1994). Flora de San Juan, República Argentina (Vol. I) Buenos Aires: Editores Vázquez-Mazzini.

Kiesling, R. (2003). Flora de San Juan, República Argentina (Vol. II) Buenos Aires: Estudio Sigma.

Kovach, W. L. (2001). Multivariate statistical package computing services. Wales: Pentrath.

Lobo, F. y Quinteros, S. (2005). A morphology-based phylogeny of Phymaturus (Iguania: Liolaemidae) with the description of four new species from Argentina. Pape is Avulsos de Zoologia, 45, 143-177.

Lobo, F., Abdala, C. y Valdecantos, S. (2012). Morphological diversity and phylogenetic relationships within a South-American clade of Iguanian lizards (Liolaemidae: Phymaturus). Zootaxa, 3315, 1-41.

Lobo, F., Laspiur, A. y Acosta, J. C. (2013). Description of new andean species of the genus Phymaturus (Iguania: Liolaemidae) from Northwestern Argentina. Zootaxa, 3683, 117-132.

Martínez-Carretero, E. (2007). Diversidad biológica y cultural de los Altos Andes Centrales de la Argentina: línea de base de la Reserva de la Biosfera San Guillermo. En E. Martínez-Carretero, A. Dalmasso, J. Márquez y G. Pastrán (Eds.), Vegetación: comunidades vegetales y unidades fitogeográficas Cap. VIII (pp. 113-152). San Juan: Universidad Nacional de San Juan.

Martori, R., Juárez, R. y Aun, L. (2002). La taxocenosis de lagartos de Achiras, Córdoba, Argentina: parámetros biológicos y estado de conservación. Revista Española de Herpetología, 16, 73-91.

O’Grady, S. P., Morando, M., Ávila, L. y Dearing, M. D. (2005). Correlating diet and digestive tract specialization: examples from the lizard family Liolaemidae. Zoology, 108, 201-210.

Olesen, J. M. y Valido, A. (2003). Lizards as pollinators and seed dispersers: an island phenomenon. Trends in Ecology and Evolution, 18, 177-181.

Perry, G. y Garland, T., Jr. (2002). Lizard home ranges revisited: effects of sex, body size, diet, habitat and phylogeny. Ecology, 83, 1870-1885.

Pianka, E. R. (1973). The structure of lizard communities. Annual Review of Ecology and Systematics, 4, 53-74.

Pinkas, L., Oliphanat, M. y Iverson, Z. (1971). Food habits of albacore bluefin tuna and bonito in California waters. pp. 1-105. Fish Bulletin (152) California: Department of Fish and Games.

Pough, F. H. (1973). Lizard energetic and diet ecology. Ecology, 54, 837-844.

Pough, F. H., Andrews, R. M., Cadle, J. E., Crump, M. L., Savitzky, A. H. y Wells, K. D. (2001). Herpetology. New Jersey: Prentice Hall. 
Quatrini, R., Albino, A. y Barg, M. (2001). Variación morfológica y dieta en dos poblaciones de Liolaemus elongatus Koslowsky, 1896 (Iguania: Tropiduridae) del noroeste patagónico. Revista Chilena de Historia Natural, 74, 639-651.

Rocha, C. F. D. (2000). Selectivity in plant food consumption in the lizard Liolaemus lutzae from Southeastern Brazil. Studies on Neotropical Fauna Environment, 35, 14-18.

Salvioli, G. (2007). Caracterización hidrometeorológica. En E. MartínezCarretero (Ed.), Diversidad biológica y cultural de los Altos Andes Centrales de la Argentina: línea de base de la reserva de la biosfera San Guillermo (pp. 63-87). San Juan: Universidad Nacional de San Juan.

Sazima, I., Sazima, C. y Sazima, M. (2005). Little dragons prefer flowers to maidens: a lizard that laps nectar and pollinates trees. Biota Neotropical, 5 , $1-8$.

Siegel, S. (1980). Estadística no paramétrica. México, D. F: Editorial Trillas.

Sokal, R. R. y Rohlf, F. J. (1999). Introducción a la bioestadística. México, D.F: Editorial Reverté, S. A.
Stevens, C. E. (1980). The gastrointestinal tract of mammals: major variations. En K. Schmidt-Nielsen, L. Bolis y C. R. Taylor (Eds.), Comparative physiology: primitive mammals (pp. 52-62). New York: Cambridge University Press.

Stevens, C. E. y Hume, D. (1998). Contributions of microbes in vertebrate gastrointestinal tract to production and conservation of nutrients. Physiological Reviews, 78, 393-427.

Valdecantos, M. S., Arias, F. y Espinoza, R. E. (2012). Herbivory in Liolaemus poecilochromus, a small, cold-climate lizard from the Andes of Argentina. Copeia, 2, 203-210.

Videla, F. (1983). Hábitos alimentarios en iguánidos del oeste árido de la Argentina. Deserta, 7, 192-202.

Villavicencio, H. J., Acosta, J. C. y Cánovas, M. G. (2005). Dieta de Liolaemus ruibali (Iguania: Liolaeminae) en la Reserva de Usos Múltiples Don Carmelo, San Juan. Argentina. Multequina, 14, 47-52.

Vitt, L. J. (2004). Shifting paradigms: herbivory and body size in lizards. Proceedings of the National Academy of Sciences, 48, 16713-16714. 